\title{
Perfil demográfico dos Hupd'äh, povo Maku da região do Alto Rio Negro, Amazonas (2000-2003)
}

\author{
Marina Machado* \\ Heloísa Pagliaro** \\ Roberto G. Baruzzi***
}

\begin{abstract}
Os Hupd'äh são um povo de língua Maku, habitante da região do Alto Rio Negro, Amazonas, Brasil. Lideranças indígenas, antropólogos, missionários e profissionais de saúde afirmam que eles estariam vivendo em condições de saúde precárias, com alta mortalidade geral e infantil. A partir de dados provenientes do Distrito Especial Indígena do Rio Negro-DSEI-RN, da Funasa/MS, para o período 2000-2003, foi realizado um estudo descritivo para avaliar o perfil demográfico e conhecer melhor essa população, contribuindo para a implementação de políticas públicas que Ihe favoreçam e para os debates em antropologia, demografia e saúde indígena. A população de 1.487 indivíduos, em 2003, cresceu 8,4\% ao ano no período estudado. Sua composição por idade e sexo indica concentração de jovens (44,9\% com menos de 15 anos), além da predominância de população do sexo masculino. A taxa bruta de natalidade (TBN) média do período foi de 33,4 nascimentos por mil habitantes, a de fecundidade total (TFT) correspondeu a 3,4 filhos por mulher, a de mortalidade (TBM) foi de 10 óbitos por mil habitantes e a de mortalidade infantil (TMI) chegou a 116,3 óbitos por mil nascimentos. $O$ aperfeiçoamento da coleta de informações, o elevado crescimento vegetativo e a intensa mobilidade espacial dos Hupd'äh poderiam explicar o alto ritmo de crescimento verificado entre 2000 e 2003.
\end{abstract}

Palavras-chave: Índios da América do Sul. Povo Hupd’äh. Povos do Rio Negro. Demografia indígena.

\section{Introdução}

Estudos demográficos sobre povos indígenas no Brasil são pouco frequentes devido à dificuldade em se obterem informações para estimar os indicadores demográficos, bem como em razão do tamanho reduzido dessas populações, que muitas vezes não atinge uma centena de indivíduos (PAGLIARO, AZEVEDO, SANTOS, 2005).
Na década de 1950, Ribeiro (1977) alertava para a possibilidade do desaparecimento dos povos indígenas no Brasil, caso se mantivessem os efeitos da depopulação provenientes das doenças adquiridas no processo de contato. No entanto, nas últimas décadas do século XX, observou-se uma reversão desse quadro pessimista, como demonstram os estudos de Gomes (1991), Flowers (1994), Azevedo (2000),

\footnotetext{
* Mestre em Epidemiologia, consultora em Saúde Reprodutiva da Organização Não-Governamental Christian Children's Fund em Angola.

** Demógrafa, doutora em Saúde Pública, Projeto Xingu, Departamento de Medicina Preventiva da Universidade Federal de São Paulo, Unifesp/EPM.

${ }_{* * \star}$ Professor titular, Projeto Xingu, Departamento de Medicina Preventiva da Universidade Federal de São Paulo, Unifesp/ EPM.
} 
Pagliaro (2002), Santos e Coimbra Júnior (2003), que revelam taxas médias de crescimento de $3 \%$ a $5 \%$ ao ano para diversos povos indígenas, enquanto a população brasileira, no período 1996-2000, cresceu $1,6 \%$ em média (IBGE, 2004).

De acordo com informações do Instituto Socioambiental (2005), existem no Brasil atualmente 220 nações indígenas e cerca de 180 línguas diferentes, além de 50 povos isolados. Segundo a Fundação Nacional do Índio (FUNAI, 2006) existiam 450 mil índios, em 2006, e o Sistema de Informação em Saúde Indígena (Siasi) contabilizava 454 mil índios, em 2005 (FUNASA, 2005). Ambas as cifras referem-se à população que vive em aldeias.

Em períodos relativamente recentes, os Hupd'äh foram objeto de estudo de vários trabalhos antropológicos. Algumas investigações fazem referências a aspectos demográficos desse povo, como duas pesquisas de Pozzobon (1983 e 1991): a primeira enfocando a relação entre o descumprimento das regras do sistema de parentesco dos povos Maku e o seu comportamento demográfico, com destaque para a discussão sobre a escassez relativa de mulheres na população (1983); e a segunda dedicada aos Maku Yohupd `ah, destacando aspectos da fecundidade dos Hupd'äh da região do Rio Tiquié.

Em estudo baseado no Censo Indígena Autônomo do Rio Negro de 1992, além de dados etnográficos, Azevedo (2003) também apresenta informações demográficas sobre os povos Maku, estimando o volume de sua população para o período de 1992-1995 e para 2002. As investigações de SilverwoodCope (1990), Reid (1979) e Athias (1995), pautadas em conhecimentos históricos e etnológicos e que muito contribuíram para a etnografia dos Hupd'äh, oferecem somente informações sobre o volume dessas populações nos momentos de suas pesquisas.

Este trabalho tem o objetivo de apresentar o perfil demográfico dos Hupd'äh, povo Maku da região do Alto Rio Negro (AM), no período 2000-2003, visando aperfeiçoar o conhecimento sobre este povo e contribuir para os debates e novas reflexões nos campos da demografia indígena e da antropologia.

\section{População}

Os Hupd'äh vivem na região interfluvial dos Rios Uaupés, Tiquié e Papuri e pertencem ao tronco linguístico Maku, com outros cinco grupos étnicos: YoHupd'äh, Kákwa, Nukak, Nadöb e Döw.

A relação entre os Hupd'äh e os povos do grupo Tukano, também habitantes da região, vem sendo descrita como de escravidão, assimétrica, simbiótica ou como de patrão-cliente (RAMOS et al., 1980). Conforme Silverwood-Cope (1990), Reid (1979), Pozzobon (1991) e Athias (1995), esses adjetivos não são capazes de exprimir com fidelidade a modalidade da relação existente entre os dois grupos, a qual seria fruto de um viés analítico provocado por informantes dos grupos Tukano.

As referências preconcebidas em relação aos Hupd'äh estendem-se à sua organização social e acervo tecnológico rudimentar, por suposto desconhecimento de técnicas agrícolas, de habilidades no uso de remo e na construção de casas comunais. $\mathrm{O}$ fato de praticarem a endogamia linguística por se casarem com pessoas do próprio tronco linguístico, traço que os diferencia em parte dos demais grupos da região, faz com que sejam considerados não-humanos pelos Tukano, que são predominantemente exogâmicos. Por conhecerem profundamente a floresta e nela se movimentarem com extrema desenvoltura, tanto durante o dia como à noite, são conhecidos, pelos Tukano, como detentores de habilidades dos animais que vivem na floresta.

\section{Material e métodos}

O estudo é do tipo descritivo de corte transversal, enfocando o período 20002003.

As fontes de informações são: 1) os censos populacionais, cadastros das famílias, censos vacinais e relatórios bimestrais e anuais de atividades das equipes de saúde do Distrito Sanitário Especial Indígena do Rio Negro (DSEl-RN), de 2000 a 2003; 2) os dados do Sistema de Informações em Saúde da Secretaria Municipal da Saúde de São Gabriel da Cachoeira, no Estado 
do Amazonas; 3) e observação direta nas comunidades Hupd'äh.

Entre as dificuldades encontradas na coleta e sistematização das informações, destacam-se os problemas relativos à sobre e subenumeração de nascimentos e a má qualidade das estimativas de idade e de classificação étnica dos indivíduos. Em relação aos nascimentos, observou-se duplicação de registros a partir de 2001, da ordem de $1 \%$ do total de nascimentos, e registros de crianças da etnia YoHupd'äh computados como sendo Hupd'äh. As correções foram feitas a partir da localidade de residência, comunidade, rio e sobrenome dos indivíduos, que coincide com as denominações de seus clãs. A maioria dos índios da região recebe nomes em português, devido à prática do batismo instituída pelas missões católicas. Entretanto, é possível reconhecer um clã pelo sobrenome, que é o mesmo para todos de um mesmo clã e identifica a etnia.

Os indicadores demográficos estimados são: volume da população; taxas de crescimento médio anual; composição por idade e sexo; razões de sexo; taxas brutas de natalidade; taxas de fecundidade geral; taxas de fecundidade total e específica por idade da mãe; taxas de mortalidade infantil; taxas brutas de mortalidade observadas e padronizadas por idade, tendo como padrão a população indígena no Brasil, de acordo com as informações oficiais da Fundação Nacional de Saúde (Funasa), de 2001.

Os dados censitários registrados pelo DSEI-RN apresentaram diferenças importantes de um ano para outro, quanto a volume da população, distribuição por idades e sexo, nascimentos e óbitos. Em relação ao grupo de menores de um ano de idade, por exemplo, observou-se que o censo de 2001 não contemplou os nascimentos ocorridos neste ano. A população registrada neste grupo etário foi de sete indivíduos e o número de nascimentos correspondeu a 42 crianças. Com o propósito de contornar essas imprecisões, foram considerados, para fins de análise, os indicadores estimados para a média do período 2000-2003, mais fidedignos do perfil populacional da população investigada.

\section{Resultados}

As informações sobre volume da população Hupd'äh, num passado recente, estão reunidas na Tabela 1 . Os dados mostram uma certa estabilidade dessa população no período 1974-2002. Conforme Bamonte (1972), na década de 1970, havia 74 povoados Hupd'äh na região, sendo 52 no Rio Tiquié, 12 na região do Médio Uaupés e dez no Rio Papuri, com uma população estimada de 1.200 indivíduos (REID, 1979). Em 1984, foram identificados 35 grupos locais, com 1.139 habitantes (ATHIAS, 1995); em 1989 havia 1.200 Hupd'äh entre os Rios Papuri e Tiquié (POZZOBON, 1991).

Conforme Azevedo (2003), o conjunto dos povos falantes de língua Maku englobava 1.203 indivíduos, em 1992, e 1.427, em 2002. Ressaltando as dificuldades em recensear os Maku, em razão da sua mobilidade espacial constante, a autora estima que, de um total de 25 comunidades identificadas e cadastradas entre 1992 e 1995, quatro deixaram de existir, nenhuma nova surgiu,

TABELA 1

População Hupd'äh do Amazonas estimada em diferentes estudos 1974-2002

\begin{tabular}{lcl}
\hline Períodos & População média estimada & Estudos \\
\hline $1974-1976$ & 1.200 & Reid (1979:15) \\
$1984-1985$ & 1.139 & Athias (1995:43) \\
$1988-1991$ & 1.210 & Pozzobon (1991:67) \\
$1992-1993$ & 1.203 & Azevedo (2003:77) \\
$1992-1995$ & 1.398 & Azevedo (2003:55-56) \\
2002 & 1.427 & Azevedo (2003:55-56) \\
\hline
\end{tabular}

Fonte: Reid (1979), Athias (1995), Pozzobon (1991) e Azevedo (2003). 
oito perderam população e 13 ganharam (AZEVEDO 2003).

No período 2000-2003, avaliado no presente estudo, a população Hupd'äh aumentou de 1.157 para 1.487 indivíduos, ou 8,4\% em média ao ano (Tabela 2 ).

Diversos fatores podem ter influenciado o crescimento populacional verificado nesse período, como a melhoria da qualidade da informação, a dupla contagem de alguns indivíduos, o ingresso de pessoas Hupd'äh na região, além do saldo vegetativo, que, avaliado isoladamente, não explicaria o crescimento observado, pois representou apenas $23 \%$ dos $8,4 \%$ ao ano que a população cresceu entre 2000 e 2003.

\section{Estrutura por idade e sexo}

As pirâmides etárias dos Hupd'äh construídas para cada ano do período 2000-2003 são bastante irregulares e refletem a oscilação acarretada pelos pequenos números (Gráfico 1).
Para contornar as irregularidades apresentadas na informação anual, construiu-se uma pirâmide baseada na composição média da população por idade e sexo no período 2000-2003.

A pirâmide resultante desse exercício, de base larga e estreitamento nas faixas etárias mais elevadas, mostra uma composição etária tipicamente jovem, além de indicar as irregularidades provocadas pelos pequenos números e a má qualidade das informações sobre as idades e o sexo (Gráfico 2).

A distribuição do povo Hupd'äh por grandes grupos etários também aponta para uma população tipicamente jovem. $\mathrm{Na}$ média do período $2000-2003,44,9 \%$ das pessoas estavam no grupo de menos de 15 anos de idade, $46,5 \%$ encontravam-se entre os adultos e 8,6\% eram indivíduos de 50 ou mais anos, considerados velhos nas sociedades indígenas (Tabela 3). A idade mediana foi de 17 anos.

As razões de sexo ao nascimento da média do período 2000-2003 (Tabela 4)

TABELA 2

Algumas características demográficas dos Hupd'äh dos Rios Tiquié e Uaupés-AM 2000-2003

\begin{tabular}{cccccc}
\hline Anos & População & Crescimento médio anual (\%) & Nascimentos & Óbitos & Saldo vegetativo \\
\hline 2000 & 1.157 & 13,7 & 13 & 13 & 0 \\
2001 & 1.327 & 1,2 & 42 & 19 & 23 \\
2002 & 1.343 & 10,2 & 32 & 13 & 19 \\
2003 & 1.487 & & & & 34 \\
\hline $\mathbf{2 0 0 0 - 2 0 0 3}$ & & $\mathbf{8 , 4}$ & 42 & 8 & $\mathbf{7 6}$ \\
\hline
\end{tabular}

Fonte: DSEI-RN, SSL, 2000 e 2001; FOIRN, 2002 e 2003.

TABELA 3

População Hupd'äh dos Rios Tiquié e Uaupés-AM, por grupos de idade 2000-2003

\begin{tabular}{|c|c|c|c|c|c|c|c|}
\hline \multirow{2}{*}{ Anos } & \multicolumn{2}{|c|}{ Menos de 15 anos } & \multicolumn{2}{|c|}{15 a 49 anos } & \multicolumn{2}{|c|}{50 anos e mais } & \multirow{2}{*}{$\begin{array}{c}\text { Total } \\
\text { N. abs. }\end{array}$} \\
\hline & N. abs. & $\%$ & N. abs. & $\%$ & N. abs. & $\%$ & \\
\hline 2000 & 518 & 44,8 & 542 & 46,8 & 97 & 8,4 & 1.157 \\
\hline 2001 & 600 & 45,2 & 615 & 46,3 & 112 & 8,4 & 1.327 \\
\hline 2002 & 622 & 46,3 & 614 & 45,7 & 107 & 8,0 & 1.343 \\
\hline 2003 & 642 & 43,2 & 701 & 47,1 & 144 & 9,7 & 1.487 \\
\hline 2000-2003 & 596 & 44,9 & 618 & 46,5 & 115 & 8,6 & \\
\hline
\end{tabular}

Fonte: DSEI-RN, SSL 2000 e 2001 e Foirn 2002 e 2003. 
GRÁFICO 1

Pirâmide etária dos Hupd'äh dos Rios Tiquié e Uaupés-AM, por sexo 2000-2003
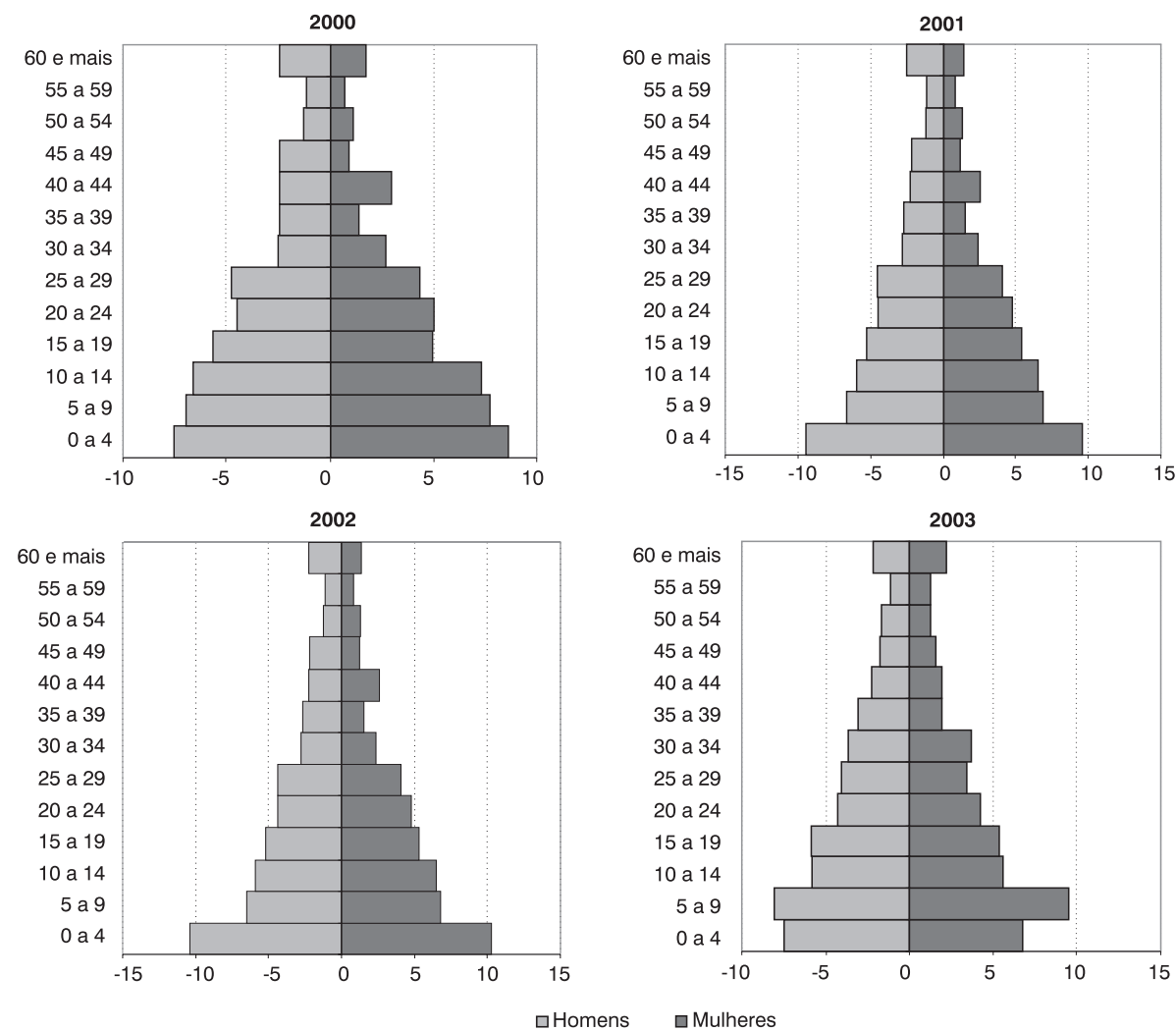

Fonte: SSL, 2000 e 2001; DSEI-Foirn, 2002 e 2003.

GRÁFICO 2

Pirâmide etária dos Hupd'äh dos Rios Tiquié e Uaupés-AM, baseada na população média do período 2000-2003

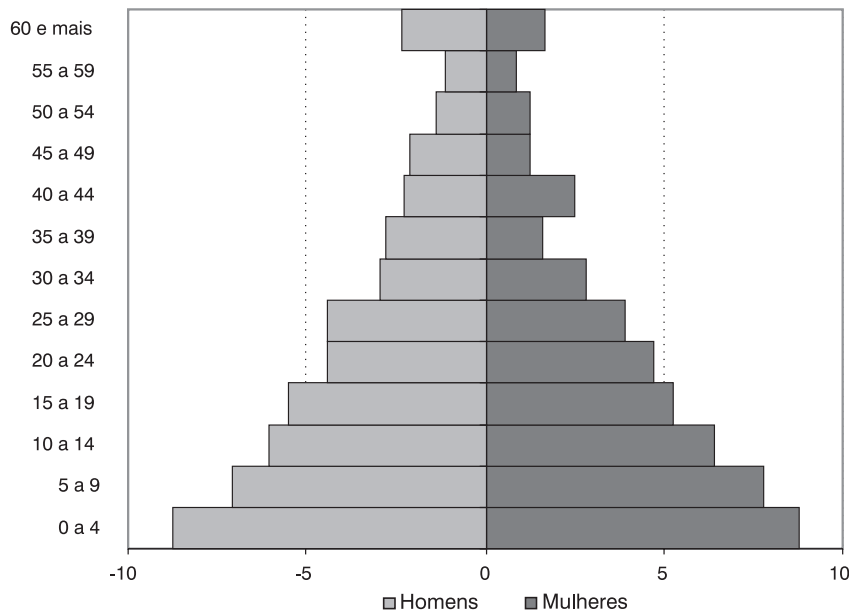

Fonte: DSEI-RN, SSL, 2000 e 2001; DSEI-RN-Foirn 2002 e 2003. 
TABELA 4

Nascimentos por sexo e razões de sexo ao nascer dos Hupd'äh dos Rios Tiquié e Uaupés-AM 2000-2003

\begin{tabular}{ccccc}
\hline \multirow{2}{*}{ Anos } & \multicolumn{2}{c}{ Nascimentos } & Total & $\begin{array}{c}\text { Razão de sexos } \\
\text { (por 100) }\end{array}$ \\
\cline { 2 - 3 } & Homens & Mulheres & & 13 \\
\hline 2000 & 7 & 6 & 42 & 116,7 \\
2001 & 23 & 19 & 32 & 121,1 \\
2002 & 17 & 15 & 42 & 113,3 \\
2003 & 16 & 26 & $\mathbf{1 2 9}$ & 61,5 \\
\hline $\mathbf{2 0 0 0 - 2 0 0 3}$ & $\mathbf{6 3}$ & $\mathbf{6 6}$ & $\mathbf{9 5 , 5}$ \\
\hline
\end{tabular}

Fonte: DSEI-RN, SSL, 2000 e 2001 e Foirn 2002 e 2003.

estariam indicando um equilíbrio entre os nascimentos de crianças dos sexos masculino e feminino ( $R S=95,5$ ). Os dados anuais mostram a predominância de nascimentos de meninos de 2000 a 2002 e de meninas em 2003. Neste caso também cabe destacar a interferência de possíveis problemas de sub e sobreregistros de nascimentos e do pequeno volume da população, que provocam a oscilação dos dados, conforme referido anteriormente na metodologia.

Para o conjunto da população, a razão de sexo estimada para os Hupd'äh foi de 103,9 homens para cada 100 mulheres, na média do período 2000-2003.

\section{Natalidade e fecundidade}

A taxa bruta de natalidade (TBN) dos Hupd'äh apresentou variações importantes entre 2000 e 2003, resultando numa média de 33,4 nascimentos vivos por mil habitantes no período (Tabela 5).

A taxa de fecundidade geral (TFG), que reporta os nascimentos vivos à população feminina em idade fértil e que, em princípio, serve para refinar a TBN, também oscilou, resultando numa média de 103,1 nascimentos por mil mulheres entre 15 e 49 anos, no período 2000-2003. Este indicador também foi sensível ao pequeno volume da população.

As taxas de fecundidade total (TFT) ${ }^{1}$ das mulheres Hupd'äh variaram entre 1,9 e 4,8 filhos por mulher, entre 2000 e 2003, com a média no período de 3,4 filhos por mulher, considerada o nível mais provável de fecundidade dessas mulheres. As variações encontradas nas taxas anuais estariam apontando, em alguma medida, a má qualidade da informação, expressa pela sub e sobreenumeração e pelo registro tardio de nascimentos. Destaca-se, ainda, o pequeno número de eventos registrados anualmente para a construção dos indicadores de fecundidade, como em 2000, por exemplo, com apenas 13 nascimentos. Esta constatação enfatiza a necessidade de trabalhar com dados agrupados para vários anos, na abordagem de populações pequenas e com dados deficientes, como as populações indígenas no Brasil.

O perfil da fecundidade por idade das mulheres Hupd'äh, na média do período, indica um padrão de fecundidade tardio, dado que a contribuição das mulheres com até 29 anos de idade para a fecundidade total foi de $51,8 \%$ e a daquelas com até 34 anos correspondeu a $74,2 \%$ (Gráfico 3). Destacam-se as irregularidades encontradas na distribuição da fecundidade, principalmente a flutuação observada nos grupos de 40 a 49 anos, que poderiam indicar a má estimativa de idades das mulheres desta faixa etária, favorecendo as mulheres de 45-49 anos. Na maioria das sociedades indígenas no Brasil contempladas com estudos de fecundidade nos últimos decênios (EARLY; PETERS, 1990;

\footnotetext{
1 TFT - expressa o número médio de filhos que uma coorte hipotética de mulheres teria ao final do período reprodutivo, na ausência de mortalidade. As TFTs foram obtidas pelo método direto.
} 
TABELA 5

Taxas brutas de natalidade, de fecundidade geral e de fecundidade total dos Hupd'äh dos Rios Tiquié e UaupésAM

2000-2003

\begin{tabular}{cccc}
\hline Anos & TBN (1) & TFG (2) & TFT (3) \\
\hline 2000 & 11,2 & 55,8 & 1,9 \\
2001 & 55,7 & 139,0 & 4,8 \\
2002 & 43,0 & 108,8 & 3,6 \\
2003 & 23,9 & 109,1 & 3,4 \\
\hline $\mathbf{2 0 0 0 - 2 0 0 3}$ & $\mathbf{3 3 , 4}$ & $\mathbf{1 0 3 , 1}$ & $\mathbf{3 , 4}$ \\
\hline
\end{tabular}

Fonte: DSEI-RN, SSL, 2000 e 2001 e Foirn, 2002 e 2003.

(1) Número de nascimentos por mil habitantes.

(2) Número de nascimentos por mil mulheres entre 15 e 49 anos

(3) Número médio de filhos por mulher.

GRÁFICO 3

Taxas de fecundidade das mulheres Hupd’äh dos Rios Tiquié e Uaupés-AM, por idade 2000-2003

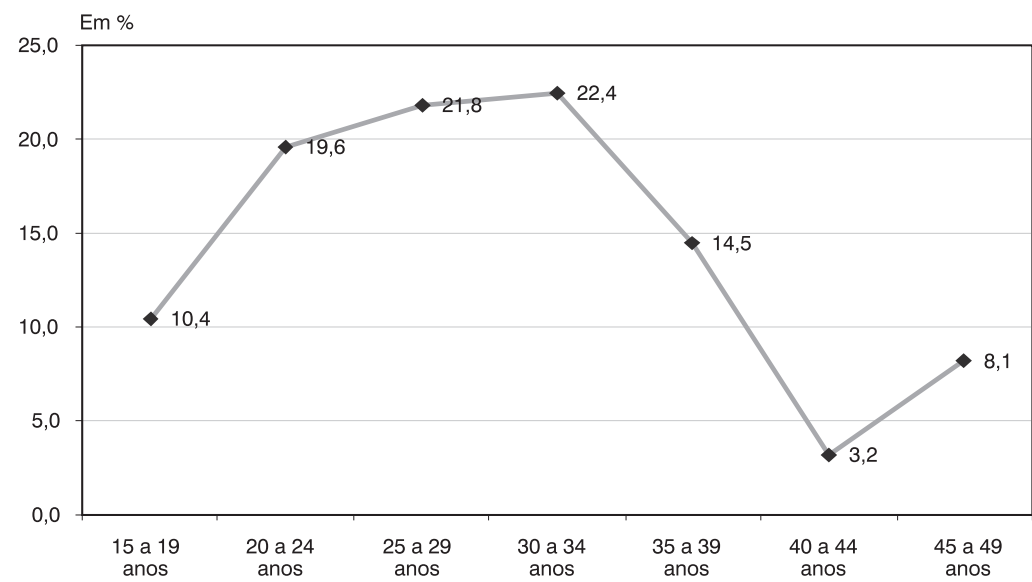

Fonte: DSEI-RN, SSL, 2000 e 2001 e Foirn, 2002 e 2003.

FLOWERS, 1994; COIMBRA et al., 2002; GOMES, 2002; AZEVEDO, 2003; PAGLIARO, 2005; PAGLIARO; JUNQUEIRA, 2007), observou-se que as mulheres encerram o período reprodutivo aos 44 anos.

\section{Mortalidade}

A taxa bruta de mortalidade (TBM) observada para o conjunto da população, na média do período 2000-2003, foi de 10 óbitos por mil habitantes, e a padronizada correspondeu a 9,2 por mil (Tabela 6).

Os níveis de mortalidade geral foram mais elevados para os homens (12,6 óbitos por mil) do que para as mulheres $(7,4$ por mil).
Quanto às taxas de mortalidade infantil (TMI), em que pese o problema, repetidamente mencionado, do tamanho reduzido da população e das dificuldades de registro sistemático das informações, que evocam distorções nos indicadores, os valores encontrados indicam a alta mortalidade infantil entre a população Hupd'äh, no período estudado. A TMI média, que procura reduzir tais distorções, atingiu o nível de 116,3 óbitos por mil nascidos vivos (Tabela 7). Vale ressaltar que, para 2003 , não foram encontrados registros de morte em menores de um ano. Optou-se, apesar disso, por descrever os resultados conforme aparecem nas informações do DSEI-Foirn, as quais são oficiais. 
TABELA 6

Taxas brutas de mortalidade observadas e padronizadas (1) dos Hupd'äh dos Rios Tiquié e Uaupés-AM, por sexo 2000-2003

\begin{tabular}{cccc}
\hline \multirow{2}{*}{ Anos } & \multicolumn{3}{c}{ TBM observada } \\
\cline { 2 - 4 } & Homens & Mulheres & Total \\
\hline 2000 & 13,7 & 8,7 & 11,2 \\
2001 & 15,0 & 13,9 & 14,3 \\
2002 & 14,6 & 4,5 & 9,7 \\
2003 & 5,7 & 3,6 & 4,7 \\
\hline 2000-2003 & $\mathbf{1 2 , 6}$ & $\mathbf{7 , 4}$ & $\mathbf{1 0}$ \\
\hline TBM padronizada & & & $\mathbf{9 , 2}$ \\
\hline
\end{tabular}

Fonte: DSEI-RN, SSL, 2000 e 2001 e Foirn, 2002 e 2003.

(1) Número de óbitos por mil habitantes.

TABELA 7

Nascidos vivos, óbitos de menores de um ano e taxas de mortalidade infantil dos Hupd'äh dos Rios Uaupés e Tiquié-AM, segundo sexo

2000-2003

\begin{tabular}{lccccc}
\hline Sexo & $\mathbf{2 0 0 0}$ & $\mathbf{2 0 0 1}$ & $\mathbf{2 0 0 2}$ & $\mathbf{2 0 0 3}$ & $\mathbf{2 0 0 0 - 2 0 0 3}$ \\
\hline $\begin{array}{l}\text { Total } \\
\text { Nascidos vivos }\end{array}$ & 13 & 42 & 32 & 42 & 129 \\
$\begin{array}{l}\text { Óbitos de menores } \\
\text { de um ano }\end{array}$ & 4 & 8 & 3 & 0 & 15 \\
TMl (1) & 307,7 & 190,5 & 93,8 & 0,0 & 116,3 \\
$\begin{array}{l}\text { Homens } \\
\text { Nascidos vivos }\end{array}$ & 7 & 23 & 17 & 16 & 63 \\
$\begin{array}{l}\text { Óbitos de menores } \\
\text { de um ano }\end{array}$ & 3 & 4 & 2 & 0 & 9 \\
$\begin{array}{l}\text { TMl (1) } \\
\text { Mulheres }\end{array}$ & 428,6 & 173,9 & 117,6 & 0,0 & 142,9 \\
$\begin{array}{l}\text { Nascidos vivos } \\
\text { Óbitos de menores } \\
\text { de um ano }\end{array}$ & 1 & 19 & 15 & 26 & 66 \\
TMl (1) & 166,7 & 4 & 1 & 0 & 6 \\
\hline
\end{tabular}

Fonte: DSEI-RN, SSL, 2000 e 2001; Foirn, 2002 e 2003.

(1) Número de óbitos de menores de um ano por mil nascidos vivos.

\section{Discussão e conclusões}

No período analisado na presente investigação (2000-2003), a população Hupd'äh cresceu $8,4 \%$ ao ano, sendo que $23 \%$ desse aumento poderia ser atribuído ao crescimento vegetativo e o restante ao ingresso de indivíduos Hupd'äh na região dos Rios Tiquié e Uaupés e à má qualidade dos dados.

Informações da história demográfica recente dos Hupd'äh, levantadas por estudiosos deste povo (REID, 1979; ATHIAS, 1995; POZZOBON, 1991; AZEVEDO, 2003), mostram que o volume dessa população variou de 1.200 a 1.427 indivíduos no período 1974-2002, equivalendo a um aumento médio próximo de $0,5 \%$ ao ano. Este ritmo de crescimento, muito inferior ao verificado em de outras populações indígenas estudadas nos últimos anos (PAGLIARO; AZEVEDO; SANTOS, 2005), poderia ser explicado, provavelmente, pelos altos níveis de mortalidade, principalmente a infantil, e níveis de fecundidade considerados moderados. Outra explicação poderia estar no fato de diferentes autores terem levantado as informações, possivelmente, por meio de diversas formas de coleta. 
Estudos demográficos realizados em populações indígenas nos últimos anos (FLOWERS, 1994; EARLY; PETERS, 1990; GOMES, 2002; PAGLIARO, 2005; SANTOS; COIMBRA Jr., 2003) confirmam que alguns grupos indígenas no país experimentaram flutuações em seus níveis de mortalidade e natalidade nas últimas décadas que, no longo prazo, resultaram em taxas de crescimento que variaram entre $3 \%$ e $5 \%$ ao ano, como os Xavánte (SOUZA; SANTOS, 2001; COIMBRA Jr. et al., 2002), os Ikpeng (MAIA et al., 2004), os Bakairi (PICCHI, 1994), os Kaiabi (PAGLIARO, 2005) e os Suyá (PAGLIARO et al., 2007).

No período 1991-2000, o conjunto da população brasileira residente na Região Norte do país cresceu 2,86\% ao ano (IBGE, 2004) e o do Estado do Amazonas, onde se localizam as comunidades Hupd'äh, aumentou 2,5\% (DATASUS, 2005).

O nível de natalidade estimado para os Hupd'äh, de 33,4 nascimentos por mil habitantes, entre 2000 e 2003, estaria bem abaixo do encontrado em outras populações indígenas da Amazônia Legal, como os Kaiabi, por exemplo, cujas TBNs variaram de 50 a 55 nascimentos vivos por mil habitantes entre 1970 e 1999 (PAGLIARO, 2005). Para os Xavante de Pimentel Barbosa, Flowers (1994) estimou uma TBN de 51,4 nascimentos vivos por mil habitantes, no período 1977-1990. Para os Xavante de Sangradouro-Volta Grande, Souza e Santos (2001) calcularam uma TBN de 57,5 por mil, entre 1993 e 1997. Para os Kamaiurá, Pagliaro e Junqueira (2007) estimaram níveis de natalidade que variaram entre 35,3 e 45,7 nascimentos por mil habitantes, no período de 1970 a 1999, que estariam mais próximos do nível encontrado entre os Hupd'äh $(33,4)$.

É importante lembrar que as informações demográficas sobre os Hupd'äh são bastante deficientes e que o nível de natalidade encontrado pode estar subestimado devido à subnotificação de nascimentos e da população considerada no denominador.
As variações observadas nas estimativas das razões de sexo ao nascer, por anos, além de refletirem o efeito dos pequenos números, podem sugerir uma hipótese que ainda carece de estudo mais aprofundado e que diz respeito à prática cultural de infanticídio nessa sociedade, indicando a seletividade por sexo. Observações de diversos estudiosos desse povo relatam que o infanticídio é prática corrente entre os Hupd'äh, quando crianças são indesejadas ou nascem defeituosas. Neste caso, os bebês são abandonados na floresta por ocasião do parto.

Há relatos ${ }^{2}$ de que as mulheres Hupd'äh praticavam o infanticídio, o que vinha sendo reprimido por parte dos missionários salesianos desde a década de 1970. Em um encontro de mulheres Hupd'äh realizado pela Associação Saúde Sem Limites (SSL), em 2005, elas relataram que ainda praticam o infanticídio, que consiste no abandono da criança recém-nascida na floresta. ${ }^{3} \mathrm{Na}$ ocasião, as mulheres não explicitaram se havia alguma relação entre o abandono e o sexo da criança.

As taxas de fecundidade global das muIheres Hupd'äh, próximas de 103 nascimentos por mil mulheres em idade reprodutiva, na média do período estudado, possuem nível similar ao da população rural do Brasil, em torno de 107 por mil (IBGE, 2004).

O nível de fecundidade estimado entre os Hupd'äh, de 3,4 filhos em média por mulher, em 2000-2003, estaria associado a padrões reprodutivos caracterizados pelo uso de práticas contraceptivas tradicionais e aborto. O infanticídio, se confirmado, contribuiria para a declaração de um menor número de filhos nascidos vivos.

Com base em informações coletadas por Silverwood-Cope (1990) entre os BaraMaku (Kakwa) e ao examinar as genealogias deste povo, Pozzobon (1983) concluiu que o número médio de filhos por família, em todas as gerações vivas, era de três. $\mathrm{O}$ autor relacionou o nível relativamente moderado de fecundidade dessas mulheres ao espectro relativamente pequeno de possibilidades

\footnotetext{
2 Comunicação pessoal de Renato Athias.

${ }^{3}$ Comunicação pessoal de Heloísa Lessa.
} 
de casamento, visto que este ocorre entre metades exogâmicas e que a proporção de mulheres núbeis pode se apresentar reduzida e interferir na reprodução biológica do grupo.

Por outro lado, o nível de fecundidade das mulheres Hupd'äh $(3,4$ filhos por mulher) encontra-se bem abaixo do observado em outras populações indígenas da Amazônia, de 5 a 9 filhos por mulher, como mostram os estudos sobre os Yanomama do Mucajai (EARLY; PETERS 1990), os Xavánte de Pimentel Barbosa (FLOWERS, 1994; COIMBRA Jr. et al., 2002), os Kaiabi (PAGLIARO, 2005), os Kamaiurá (PAGLIARO; JUNQUEIRA, 2007), os Sateré-Mawé (TEIXEIRA; BRASIL, 2005) e alguns povos da região do Rio Negro (AZEVEDO, 2003).

Comparada ao conjunto da população brasileira, a fecundidade dos Hupd'äh seria um pouco mais elevada do que a registrada na década de 1990 (2,85 filhos nascidos vivos por mulher). Na Região Norte, onde habitam os Hupd'äh, a taxa de fecundidade total era de 3,09 filhos em 2000 (DATASUS, 2005) e a da população autodeclarada indígena no Censo Demográfico de 2000 correspondia a 5,7 filhos por mulher residente nas áreas rurais do país e de 6,2 filhos nas áreas denominadas rural específico, onde se localizam Terras Indígenas (IBGE, 2005; WONG et al., 2006).

No tocante ao perfil etário da fecundidade das mulheres Hupd ‘äh, verificou-se que $74,2 \%$ da fecundidade total seria de responsabilidade daquelas com até 34 anos, sugerindo uma fecundidade de padrão relativamente jovem se comparada à de outros povos indígenas já estudados no país. Entre as mulheres Kaiabi do Xingu, que controlam a fecundidade por métodos tradicionais e cuja TFT no período 1990-99 era de 9,5 filhos por mulher, apenas $58 \%$ da fecundidade total foi contribuição daquelas com até 34 anos de idade (PAGLIARO, 2002).

A composição por idades evidencia que a população Hupd'äh é extremamente jovem e tem convivido com altos níveis de mortalidade e fecundidade moderada. Apesar de não ter sido possível estimar o saldo migratório dessa população, sabe-se que os Hupd'äh se deslocam muito. De acordo com Athias
(1995), há evidências de relações de troca entre os Hupd'äh e os Kakwa (Bara-Maku) que vivem na Colômbia, assim como a presença de indivíduos Hupd'äh no território dos Kakwa. Alguns Hupd'äh, por vezes, prestam serviços ao povo Tukano, que vive em São Gabriel da Cachoeira, e costumam passar semanas ou meses nesta cidade.

Além do efeito das variáveis demográficas, a estrutura etária dos Hupd'äh, no período analisado, sofre os efeitos de informações deficientes, principalmente em relação aos erros de declaração de idades.

A razão de sexo estimada dos Hupd'äh, de 103,9 homens para cada 100 mulheres no conjunto da população, na média do período 2000-2003, estaria de acordo com as estimativas de Azevedo (2003), que indicam uma superioridade numérica da população masculina em todos os povos das sub-regiões do Alto Rio Negro. Conforme a autora, isto poderia indicar a preferência destes povos por filhos homens, bem como uma maior mortalidade feminina, fatos que ainda merecem uma investigação mais apurada. Por outro lado, conforme já mencionado, Pozzobon (1983) alerta para o fato de as regras de casamento variarem entre os povos Maku devido à relativa escassez de mulheres provocada pelas interdições do sistema de parentesco, o que levaria à redução do número de mulheres com as quais um homem poderia se casar.

A razão de sexos dos Hupd'äh $(103,9)$ seria superior à do conjunto da população brasileira, em 2000 (96,9 homens para cada 100 mulheres), mais próxima da verificada para o Estado do Amazonas, no mesmo ano, de 101 homens para cada 100 mulheres (DATASUS, 2005), e igual à dos SateréMawé, povo indígena localizado naquele estado e cuja razão de sexo, em 2003, era de 103,3 homens para cada 100 mulheres (TEIXEIRA; BRASIL, 2005).

A análise das taxas de mortalidade geral dos Hupd'äh, como dos demais indicadores demográficos, deve considerar também a má qualidade das informações utilizadas, neste caso no sentido de subnotificação dos óbitos e das estimativas populacionais consideradas nos denominadores. O nível de mortalidade geral padronizado encontrado 
entre os Hupd'äh seria próximo de 10 óbitos por mil habitantes, entre 2000 e 2003. A taxa de mortalidade infantil entre os Hupd'äh, no período estudado, era de 116,3 óbitos por mil nascidos vivos.

Em análise das causas de morte de menores de um ano, Machado (2006), que avalia o perfil de mortalidade dos Hupd' äh, mostra que, dos 15 registros de óbitos neste grupo etário, seis (40\%) foram por doenças infecciosas e parasitárias (diarréias e septicemia), cinco (33\%) por doenças do aparelho respiratório (pneumonias), três (20\%) por causas indeterminadas, havendo o registro de um caso $(7 \%)$ de malformação congênita. De acordo com a pesquisa de Oliveira e Mendes (1999) sobre a evolução da mortalidade infantil no Brasil, os Hupd'äh apresentam indicadores semelhantes aos da década de 1960 no país.

Os resultados encontrados entre os Hupd'äh superam os achados de Melchior et al. (2002), Garnelo et al. (2005) e Cardoso et al. (2005), que estudaram outros povos indígenas e confirmam a necessidade de medidas de intervenção que reduzam esse indicador por meio de melhorias no acesso aos serviços de saúde.

A realização desta pesquisa entre os Hupd'äh também evidenciou as dificuldades enfrentadas pelo Distrito Sanitário Especial Indígena do Rio Negro - DSEI-RN quanto à manutenção de um sistema de informação ágil, não fragmentado e de fácil acesso para subsidiar as equipes de saúde. Apesar das dificuldades apontadas, os DSEls apresentam-se como o melhor espaço para a coleta das informações sobre a saúde indígena, no estágio atual da execução da Política Nacional de Saúde para os Povos Indígenas, isto devido aos problemas de disponibilidade de informações no nível central da Funasa.

\section{Referências}

ATHIAS, R. Hupdë-Maku et Tukano - Les rélations inégales chex deux societés du Uaupés Amazonien, Brésil. Tese. (Doutorado). Paris: Université Paris X (Natèrre), 1995.

AZEVEDO, M. M. Censos demográficos e os "índios": dificuldades para reconhecer e contar. In: RICARDO, C. A. (Org.). Povos
É conhecida a dificuldade dos profissionais de saúde em preencher formulários de informações, principalmente porque, na maioria dos casos, isso decorre das imposições do serviço que não oferece espaços de reflexão sobre a importância dessas informações para racionalizar as ações, para que os dados se transformem em instrumentos de diálogo, mobilização comunitária e negociação política, visando a melhoria dos serviços e das condições de trabalho.

Assim, seria um interessante exercício para os profissionais de saúde, apoiados por antropólogos, linguistas, educadores e demógrafos, a criação de espaços para discussão com os Hupd'äh sobre, por exemplo, como contá-los, haja vista sua alta mobilidade, e para quê contá-los; como são tradicionalmente registrados os nascimentos; como são acompanhados os indivíduos em processo de morte; como as mulheres e homens esperam seus filhos; qual o número adequado de filhos; que cuidados têm na gestação, no parto e no pós-parto. Essas discussões poderiam servir de base para estudos etno-demográficos que subsidiariam o planejamento de ações de saúde, considerando os conhecimentos da população.

A dinâmica demográfica expressa as transformações históricas em uma dada sociedade, as opções de indivíduos e famílias, o contexto social e o saber da população, auxiliando no planejamento dos serviços de saúde com base nessas informações. Os Hupd'äh dominam o seu território e o que nele acontece; e, assim como criam inúmeras vias de interligação na floresta, certamente poderão indicar rumos para a abertura de caminhos no sentido da promoção da saúde e da melhoria das formas de coleta de informação.

indígenas do Brasil 1996/2000. São Paulo: Instituto Socioambiental, 2000, p. 79-83.

Demografia dos povos indígenas do Alto Rio Negro/AM: um estudo de caso de nupcialidade e reprodução. Tese (Doutorado). Campinas: Universidade Estadual de Campinas, 2003. 
BAMONTE, G. Localização de aldeias Macus na área do Rio Tiquié. In: 40 CONGRESSO INTERNAZIONALE DEGLI AMERICANISTI. Roma, Itália, 3-10 settembre, 1972 [estratto p. 439-447].

CARDOSO, A. M.; SANTOS, R. V.; COIMBRA Jr., C. E. A. Mortalidade infantil segundo raça/cor no Brasil: o que dizem os sistemas nacionais de informação? Cadernos de Saúde Pública, 21(5):1.602-1.608, 2005.

COIMBRA Jr., C. E. A.; FLOWERS, N. M.; SALZANO, M. F.; SANTOS, R. V. The Xavánte in transition: health, ecology and bioantrhopology in Central Brasil. Ann Arbor: University of Michigan Press, 2002.

DATASUS, 2005. Disponível em: < http:// tabnet.datasus.gov.br $>$.

EARLY, J. D.; PETERS, J. F. The population dynamics of the Mucajaí Yanomama. New York: Academic Press, 1990.

FLOWERS, N. M. Crise e recuperação demográfica: os Xavante de Pimentel Barbosa, Mato Grosso. In: SANTOS, R. V.; COIMBRA Jr., C. E. A. (Org.). Saúde dos povos indígenas. Rio de Janeiro: Fiocruz, 1994, p. 213-242.

FUNAl, 2005. Disponível em: < http://www. funai.gov.br/indios/fr_conteudo.htm $>$.

FUNASA, 2005. Disponível em: <https:// sis.funasa.gov.br/portal/detalhe_dsei. asp?blnbrasil $=$ true $>$.

GARNELO, L.; BRANDÃO, L. C.; LEVINO, A. Dimensões e potencialidades dos sistemas de informação geográfica na saúde indígena. Rev. Saúde Pública, 39(4):634640, 2005.

GOMES, M. P. Os índios e o Brasil. Ensaio sobre um holocausto e sobre uma nova possibilidade de convivência. $2^{\mathrm{a}}$ ed. Petrópolis: Vozes, 1991.

0 índio na história. $O$ povo Tenetehara em busca da liberdade. Petrópolis: Editora Vozes, 2002.

IBGE - Instituto Brasileiro de Geografia e Estatística. Tendências demográficas. Uma análise dos resultados da amostra do
Censo Demográfico 2000. Rio de Janeiro, 2004 (Coleção Estudos \& Pesquisas, 13).

Tendências demográficas. Uma análise dos indígenas com base nos resultados da amostra dos Censos Demográficos 1991 e 2000. Rio de Janeiro, 2005.

INSTITUTO SOCIOAMBIENTAL, 2005. Disponível em: <http://www.socioambiental. org/pib/portugues/quonqua/qoqindex. htm $>$.

MELCHIOR, S. C.; SOARES, D. A.; ANDRADE, S. M.; IZUMI, R. M. K. Avaliação da mortalidade de grupos indígenas do norte do Paraná. Informe Epidemiológico do SUS, 11(2):61-68, 2002.

OLIVEIRA, L. A. P.; MENDES, M. M. S. Mortalidade infantil no Brasil: tendências recentes. In: MINAYO, M. C. S. (Org.). Os muitos Brasis: saúde e população na década de 80. 2ª ed. São Paulo: Hucitec; Rio de Janeiro: Abrasco, 1999, p 291-303.

PAGLIARO, H.; CARVALHO, N. S.; RODRIGUES, D.; BARUZZI, R. G. Dinâmica demográfica dos Suyá, povo Jê do Parque Indígena do Xingu. Cadernos de Saúde Pública, v. 23, n. 5, p. 1.071-1.081, maio 2007.

PAGLIARO, H.; JUNQUEIRA, C. Recuperação populacional e fecundidade dos Kamaiurá, povo tupi do Parque Indígena do Xingu, Brasil Central, 1970-2003. Saúde e Sociedade, v. 16 , n. 2, p. 37-47, agosto 2007.

PAGLIARO, H. A revolução demográfica dos povos indígenas: a experiência dos Kaiabi do Parque Indígena do Xingu, Mato Grosso. In: PAGLIARO, H.; AZEVEDO, M. M.; SANTOS, R. V. (Org.). Demografia dos povos indígenas no Brasil. Rio de Janeiro: Editora Fiocruz/Abep, 2005, p. 79-102.

PAGLIARO, H.; AZEVEDO, M. M.; SANTOS, R. V. Demografia dos povos indígenas no Brasil: um panorama crítico. In: PAGLIARO, H.; AZEVEDO, M. M.; SANTOS, R. V. (Org.). Demografia dos povos indígenas no Brasil. Rio de Janeiro: Fiocruz/Abep, 2005, p. 11-32. 
PAGLIARO, H. A revolução demográfica dos povos indígenas: a experiência dos Kaiabi do Parque Indígena do Xingu, Mato Grosso (1970-1999). Tese (Doutorado). São Paulo: Faculdade de Saúde Pública, Universidade de São Paulo, 2002.

POZZOBON, J. A. H. Isolamento e endogamia: observações sobre a organização social dos índios Maku. Dissertação (Mestrado). Porto Alegre: Instituto de Filosofia e Ciências Humanas, Universidade Federal do Rio Grande do Sul, 1983.

Parente et demographie chez Ies indiens Maku. Tese (Doutorado). Paris: U.F. D'Ethnologie, Anthropologie et Science des Religions, Université de Paris VII, 1991.

RAMOS, A. R.; SILVERWOOD-COPE, P.; OLIVEIRA, A. G. Patrões e clientes: relações intertribais no Alto Rio Negro. Hierarquia e simbiose. São Paulo: Hucitec, 1980, p.135-182.

REID, H. Some aspects of movement, growth and change among the Hupdu Maku Indians of Brazil. Tese (Doutorado). Cambridge: Faculty of Archaeology and Anthropology, University of Cambridge, 1979.

RIBEIRO, D. Os índios e a civilização: a integração das populações indígenas bra- sileiras no Brasil moderno. 2ª ed. Petrópolis: Vozes, 1977.

SANTOS, R. V.; COIMBRA Jr., C. E. A. Cenários e tendências da saúde e da epidemiologia dos povos indígenas do Brasil. In: COIMBRA Jr., C. E. A.; SANTOS, R. V.; ESCOBAR, A. L. (Org.). Epidemiologia e saúde dos povos indígenas no Brasil. Rio de Janeiro: Fiocruz/ Abrasco, 2003, p.13-47.

SILVERWOOD-COPE, P. L. Os Maku: povo caçador do noroeste da Amazônia. Brasília: Editora UnB, 1990.

SOUZA, L. G.; SANTOS, R. V. Perfil demográfico da população indígena Xavante de Sangradouro-Volta Grande, Mato Grosso (1993-1997), Brasil. Cad. Saúde Pública, 17(2):355-366, 2001.

TEIXEIRA, P.; BRASIL, M. Estudo demográfico dos Sateré-Mawé: um exemplo de censo participativo. In: PAGLIARO. H.; AZEVEDO, M. M.; SANTOS, R. V. (Org.). Demografia dos povos indígenas no Brasil. Rio de Janeiro: Fiocruz/Abep, 2005, p.135-154.

WONG, L. R.; MORELL, M. G.G.; CARVALHO, R. L. Notas sobre o comportamento reprodutivo dos povos indígenas no período 1991-2000. In: ENCONTRO NACIONAL DE ESTUDOS POPULACIONAIS, XV. Anais... Caxambu: Abep 2006. Disponível em: $<$ http://www.abep.org.br>.

\section{Resumen}

Perfil demográfico de los Hupd'äh, pueblo Maku de la región del Alto Rio Negro, Amazonas (2000-2003)

Los Hupd'äh son un pueblo de lengua Maku, habitante de la región del Alto Río Negro, Amazonas, Brasil. Liderazgos indígenas, antropólogos, misionarios y profesionales de la salud, afirman que estarían viviendo en condiciones de salud precarias, con alta mortalidad general e infantil. A partir de datos provenientes del Distrito Especial Indígena del Río Negro - DSEI-RN, de la Funasa/MS, para el período 2000-2003, fue realizado un estudio descriptivo para evaluar el perfil demográfico y conocer mejor esta población, contribuyendo con la implementación de políticas públicas que la favorezcan y para los debates en antropología, demografía y salud indígena. La población de 1.487 individuos, en 2003 , creció un $8,4 \%$ al año en el período estudiado. Su composición por edad y sexo indica concentración de jóvenes (44,9\% con menos de 15 años), además de la predominancia de población del sexo masculino. La tasa bruta de 
natalidad (TBN) media del período fue de 33,4 nacimientos por mil habitantes, la de fecundidad total (TFT) correspondió a 3,4 hijos por mujer, la de mortalidad (TBM) fue de 10 muertes por mil habitantes y la de mortalidad infantil (TMI) llegó a 116,3 muertes por mil nacimientos. El perfeccionamiento de la recolección de informaciones, el elevado crecimiento vegetativo y la intensa movilidad espacial de los Hupd'äh podrían explicar el alto ritmo de crecimiento verificado entre 2000 y 2003.

Palabras-clave: Indios de América del Sur. Pueblo Hupd'äh. Pueblos del Río Negro. Demografía indígena.

\begin{abstract}
Demographic profile of the Hupd'äh, a Maku people living the Upper Rio Negro Region, State of Amazonas, Brazil (2000-2003)

The Hupd'äh are a people of the Maku language who live in the Upper Rio Negro Region in Amazonas, Brazil. Indigenous leaders, anthropologists, missionaries and health professionals all say that this population lives under very precarious conditions of health, with high overall and child mortality rates. On the basis of data derived from the Special Indigenous District of Rio Negro (DSEI-RN, of Funasa/MS), for the period of 2000-2003, a descriptive study was conducted to evaluate the demographic and general profiles of the group The study also had the objective of contributing to the implementation of public policies for them, and for debates in the areas of anthropology, demography and indigenous health. The population of 1,487 individuals in 2003 grew $8.4 \%$ per year during the period studied. Composition by age and sex indicates a concentration of young people (44.9\% under the age of 15), and a greater percentage of males. The average gross fertility rate (TBN) for the period was 33.4 births per 1000 inhabitants, and total fertility (TFT) was 3.4 children per woman. Mortality rate (TBM) was 10 deaths per 1000 inhabitants and child mortality rate (TMI) was high, 116.3 deaths per 1000 births. More refined processes of collecting information, the high vegetative growth and the intense spatial mobility of the Hupd'äh may explain the high growth rate seen between 2000 and 2003.
\end{abstract}

Keywords: South American Indians. Hupd'äh People. Peoples of Rio Negro. Indigenous demography. 